\title{
Correction to: "Is human classification by experienced untrained observers a gold standard in fixation detection?"
}

\author{
Ignace T. C. Hooge ${ }^{1} \cdot$ Diederick C. Niehorster ${ }^{2,3} \cdot$ Marcus Nyström $^{2} \cdot$ Richard Andersson $^{4,5} \cdot$ Roy S. Hessels ${ }^{1,6}$ \\ Published online: 10 February 2021 \\ (C) The Psychonomic Society, Inc. 2021
}

\section{Correction to: Behav Res https://doi.org/10.3758/s13428-017-0955-x}

We were informed by Friedman (2020) about potential errors in Hooge et al. (2018). Indeed, we detected an error in our paper (Hooge et al., 2018). Cause of the error: 14 of the 70 (eye-tracking data) stimulus files started with missing data. Prior to the fixation-classification sessions by expert human coders, the missing data at the beginning of these 14 stimulus files were removed. Unfortunately, we forgot to replace the original stimulus files (with missing data at the start) by the new files (without missing data at the start) in the analysis and the online data set. As a result, in the classified fixations of 14 stimuli, the onset and offset times of the human coder settings were shifted.

Resulting changes: We have corrected the time shifts in the coded fixation onsets and offsets and re-analyzed the data. The following reported values in the manuscript have changed:

The online version of the original article can be found at https://doi.org/ 10.3758/s13428-017-0955-x

Ignace T. C. Hooge

i.hooge@uu.nl

1 Experimental Psychology, Helmholtz Institute, Utrecht University, Heidelberglaan 1, 3584, CS Utrecht, The Netherlands

2 Lund University Humanities Lab, Lund University, Helgonabacken 12, 22362 Lund, Sweden

3 Department of Psychology, Lund University, Lund, Sweden

4 Eye Information Group, IT University of Copenhagen, Copenhagen, Denmark

5 Department of Philosophy and Cognitive Sciences, Lund University, Lund, Sweden

6 Developmental Psychology, Utrecht University, Heidelberglaan 1, CS 3584 Utrecht, The Netherlands
1. Page 1870. "The mean amplitude ranges from $5.7^{\circ}$ (P.Z.) to $6.6^{\circ}$ (M.S.)..." changed to "The mean amplitude ranges from $5.7^{\circ}$ (P.Z.) to $6.7^{\circ}$ (M.S.)..."

2. Page 1871. "Figure 6 shows the proportions of fixations without data loss; this proportion ranges from .91 (I.H.) to .96 (T.C.)" changed to "Figure 6 shows the proportions of fixations without data loss; this proportion ranges from .93 (I.H.) to .99 (K.H.).”

3. Page 1871. “... mean number of lost samples per fixation containing data loss ranges from 21.5 (P.Z.) to 33.1 (M.S.)" changed to "... mean number of lost samples per fixation containing data loss ranges from 4.8 (K.H.) to 9.2 (M.S.)."

4. Page 1873. "... and the lowest value for M.S. $(\lambda=1.5)$ " changed to "... and the lowest value for J.F. $(\lambda=1.6)$."

We further found one typo: page 1868. “... ranged from $1.00 \mathrm{~s}$ (R.H.) to 3.37 s (R.H.) (Fig. 2b)," changed to “... ranged from $1.00 \mathrm{~s}$ (R.H.) to $3.37 \mathrm{~s}$ (R.A.) (Fig. 2b)."

The error led to negligible changes in four figures and substantial changes in one figure. The affected figures and the coder settings with corrected timings can be downloaded from https://doi.org/10.5281/zenodo.818784

Impact: The conclusions of our study were not affected. They remain as follows:

1. Human classification is not the gold standard in fixation detection.

2. Human classification is still important in algorithm validation.

3. Human classification can be useful in detecting features of the eye-tracker signal that are ill-defined.

\section{References}

Hooge, I. T. C., Niehorster, D. C., Nyström, M., Andersson, R., Hessels, R. S. (2018). Is human classification by experienced 
untrained observers a gold standard in fixation detection? Behav. Res. Methods 140, 1-18.

Friedman, L. (2020). A re-examination of the eye movement data used by

Hooge et al (2018) Is human classification by experienced untrained observers a gold standard in fixation detection? arXiv: 2001.07701v2 [q-bio.QM] 1 Apr 2020
Publisher's note Springer Nature remains neutral with regard to jurisdictional claims in published maps and institutional affiliations. 